Pacific Journal of Mathematics

ON THE LIMITING DISTRIBUTION OF ADDITIVE
FUNCTIONS (MOD 1) 


\title{
ON THE LIMITING DISTRIBUTION OF ADDITIVE FUNCTIONS (MOD 1)
}

\author{
P.D.T. A. ElliotT
}

A function $f(n)$, defined on the positive rational integers, is said to be additive if and only if for every pair of coprime integers $a$ and $b$ the relation

$$
f(a b)=f(a)+f(b)
$$

is satisfied. Thus an additive function is determined by its values on those integers which are prime powers. In an extensive paper Erdos raised the question of characterising those real valued additive functions which have a limiting distribution $(\bmod 1)$.

It is our present purpose to give such a characterisation.

He proved, in particular, that an additive function $f(n)$ is certainly uniformly distributed in the sense of Weyl if $f(p) \rightarrow 0$ as $p \rightarrow \infty$, and if the series

$$
\Sigma \frac{f^{2}(p)}{p}
$$

diverges.

For the remainder of this paper we understand a distribution function $F(z)(\bmod 1)$, or more shortly a distribution function, to have the properties

(i) $F(z)$ is increasing in the wide sense

(ii) $F(z)=F(z+)$ for all values of $z$, that is $F(z)$ is right continuous.

(iii) $F(z)=0$ if $z<0$, and $=1$ if $z \geqq 1$.

We say that a sequence of distribution functions $F_{n}(z), n=1,2, \cdots$ has a limiting distribution $(\bmod 1)$ if and only if there exists a function $F(z)$, satisfying the above three conditions, so that at every pair of points of continuity $(\alpha, \beta)$ of $F(z), 0<\alpha<\beta<1$, we have

$$
F_{n}(\beta)-F_{n}(\alpha) \rightarrow(F(\beta)-F(\alpha)), \quad(n \rightarrow \infty) .
$$

We notice that in the range $0<z<1$ any such limiting distribution $F(z)$ is determined only up to an additive constant. When the function $F(z)$ is 


$$
F(z)=\left\{\begin{array}{l}
1, z \geqq 1, \\
z, 0<z<1, \\
0, z \leqq 0,
\end{array}\right.
$$

this definition coincides with Weyl's definition [7] of uniform distribution $(\bmod 1)$.

We shall say that the sequence of real numbers $x_{1}, x_{2}, \ldots$ has a limiting distribution (mod 1) if and only if the sequence of distribution functions defined by

$$
F_{n}(z)=n^{-1} \sum_{\substack{j=1 \\ x_{j} \leqq z(\bmod 1)}}^{n} 1, \quad n=1,2, \cdots
$$

for $0 \leqq z<1$, and extended in the obvious way outside this interval, have a limiting distribution in the above sense.

In what follows, for each real number $\alpha$ we denote by $\{\alpha\}$ the fractional part of $\alpha$, that is the least positive representative of the residue class $\alpha(\bmod 1)$; and by $\|\alpha\|$ the distance of $\alpha$ from the nearest integer. Thus we have

$$
\|\alpha\|=\min (\{\alpha\}, 1-\{\alpha\}) .
$$

we shall also have occasion to use the function

$$
\operatorname{Sign} y=\left\{\begin{array}{r}
1 \text { if } y>0, \\
0 \text { if } y=0, \\
-1 \text { if } y<0 .
\end{array}\right.
$$

With these definitions, and the above meaning of limiting distribution, we can now state:

THEOREM 1. A real valued additive number theoretic function $f(n)$ has a limiting distribution $(\bmod 1)$ if and only if for each integer $\nu$ one of the following three conditions is satisfied:

(i) For each real value of the series

$$
\sum_{p} p^{-1}\|f(p)-t \log p\|^{2}
$$

is divergent.

(ii) For each positive integer $r, \nu f\left(2^{r}\right)$ is half an odd rational integer.

(iii) Both of the series

$$
\sum_{p} p^{-1}\|\nu f(p)\|^{2}, \sum_{p} p^{-1}\|\nu f(p)\| \operatorname{Sign}\left(\frac{1}{2}-\{\nu f(p)\}\right)
$$

are convergent. 
In particular $f(n)$ is uniformly distributed $(\bmod 1)$ if and only if at least one of the first two conditions is satisfied for each integer.

THEOREM 2. For each integer $\nu$ set

$$
\varepsilon_{\nu}=\left|\left(1+2^{-1} e^{2 \pi i \nu f(2)}+2^{-2} e^{2 \pi i \nu f\left(2^{2}\right)}+\cdots\right)\right| \cdot
$$

Then a limiting distribution $(\bmod 1)$ for the function $f(n)$ is

(a) Continuous if and only if

$$
N^{-1} \sum_{\nu \leq N} \varepsilon_{\nu} \exp \left(-2 \sum_{p} \sin ^{2} \pi \nu f(p)\right) \rightarrow 0, \quad(N \rightarrow \infty) .
$$

(b) Absolutely continuous with a derivative that belongs to the Lebesgue class $L^{2}[0,1]$ if and only if the series

$$
\sum_{\nu=-\infty}^{\infty} \varepsilon_{\nu}^{2} \exp \left(-4 \sum_{p} \sin ^{2} \pi \nu f(p)\right)
$$

is convergent.

In the statement of this theorem is to be understood that if a series

$$
\sum_{p} p^{-1} \sin ^{2} \pi \nu f(p)
$$

diverges, then the corresponding number

$$
\exp \left(-2 \sum_{p} \cdots\right)
$$

si defined to be zero.

We note that in either of the circumstances (a) or (b) of Theorem 2 we can assert that there exists a distribution $F(z)$ so that

$$
n^{-1} \sum_{f(n) \leqq z} 1 \rightarrow F(z), \quad(h \rightarrow \infty),
$$

holds for every real value of $z$.

For the proofs of these theorems we need essentially two lemmas. Before stating the first of these we discuss some results of Halász [4].

A number theoretic function $g(n)$ is said to be multiplicative if for every pair of coprime integers $a, b$, the relation

$$
g(a b)=g(a) g(b)
$$

is satisfied. In his paper of 1968 Halász gives necessary and sufficient criteria that multiplicative functions of wide classes have mean-value theorems of the type 


$$
\lim _{n \rightarrow \infty} n^{-1} \sum_{m \leqq n} g(m) \quad \text { exists . }
$$

It is convenient to restate some of his results. We shall adopt for the moment the notation of his paper [4], save that in place of $f(n)$ we set $g(n), n=1, \cdots$ For a fixed value of $P(\geqq 3)$ we define a multiplicative function $g^{*}(n)$ by

$$
g^{*}\left(p^{k}\right)= \begin{cases}0 & \text { for } p \leqq P, k=1,2, \cdots \\ (g(p))^{k} & \text { for } p>P, k=1,2, \cdots\end{cases}
$$

We note here that no essential use is made of the size of $P$ during any of the proofs of the theorems in Halász' paper, it being a parameter introduced as a technical convenience to ensure the nonvanishing of certain products (see pp. 369-370 of [4]). We shall also need the function

$$
\lambda(n)=\frac{1}{k}\left\{\begin{array}{l}
\text { if } n=p^{k}, p \text { prime, } k=1,2, \cdots \\
0 \text { otherwise. }
\end{array}\right.
$$

If now $g(n)$ is assumed to satisfy the inequality $|g(n)| \leqq 1$ for every integer $n$, then as Theorem 2 of his paper Halász proves that

$$
x^{-1} \sum_{m \leqq x} g(m)=C_{0} \frac{H\left(1+i a_{0}\right)}{1+i a_{0}} L_{0}(\log x) x^{1+i a_{0}}+o(x), \quad(x \rightarrow \infty),
$$

with the following understanding:

If for every value of $t$ the series

$$
\sum_{n=1}^{\infty} n^{-1} \lambda(n)\left(1-R e g^{*}(n) n^{-i t}\right)
$$

diverges ([4] p. 380), or if

$$
\left(1+g(2) 2^{-1}+g\left(2^{2}\right) 2^{-2}+\cdots\right)=0
$$

([4] p. 369) then $C_{0} L_{0}(\log x)$ is to be replaced by zero.

On the other hand, if for some values of $t$ (which is in fact unique) the above series converges, then we set $a_{0}=t$, and have ([4] p. 382),

$$
C_{0}=\exp \left(-\sum_{n=1}^{\infty} n^{-1} \lambda(x)\left(1-R e g^{*}(n) n^{-i t}\right)\right) .
$$

The function $L_{0}(\log x)$ is defined by

$$
L_{0}\left(\frac{1}{\sigma-1}\right)=\exp \left(i \sum_{n=1}^{\infty} \frac{\lambda(n)}{n^{\sigma}} \operatorname{Im}\left(g^{*}(n) n^{-i t}\right)\right), \quad \sigma>1,
$$

so that as $\sigma \rightarrow 1+$; 


$$
C_{0} L_{0}\left(\frac{1}{\sigma-1}\right) \sim \exp \left(-\sum_{n=1}^{\infty} \frac{\lambda(n)}{n^{\sigma}}\left(1-g^{*}(n) n^{-i t}\right)\right) .
$$

Here $H(s)$ is the function defined for complex numbers $s$ by

$$
H(s)=\prod_{p}\left(1+\frac{g(p)}{p^{s}}+\frac{g\left(p^{2}\right)}{p^{2 s}}+\cdots\right) \prod_{p>P}\left(1-\frac{g(p)}{p^{s}}\right),
$$

which is absolutely convergent for $\sigma=\operatorname{Re} s \geqq 1$.

Finally, we need the fact, also proved in [4], that $L_{0}(u)$ is a slowly oscillating function. In other words, $\left|L_{0}(u)\right|=1$ for all values of $u>0$, and

$$
\frac{L_{0}(y)}{L_{0}(u)} \rightarrow 1
$$

holds uniformly for $u<y \leqq 2 u$, as $u \rightarrow \infty$.

We can now state our first lemma.

Lemma 1. Let $g(n)$ be a complex valued multiplicative number theoretical function which satisfies

$$
|g(n)| \leqq 1, \quad(n=1,2, \cdots) .
$$

Then

$$
\lim _{n \rightarrow \infty} n^{-1} \sum_{m \leqq n} g(m)=C
$$

exists under the following circumstances:

(i) with $C=0$ :

Either

$$
-1=g(2)=g\left(2^{2}\right)=\cdots
$$

Or, the series

$$
\sum_{p} p^{-1}\left(1-\operatorname{Re} g(p) p^{-i t}\right)
$$

diverges for each real value of $t$.

The series

(ii) with $C \neq 0$ : 
Proof. If for any positive integer $r$, Re $g\left(2^{r}\right)>-1$, then

$$
\operatorname{Re}\left(1+\sum_{m=1}^{\infty} 2^{-m} g\left(2^{m}\right)\right)>1-\sum_{m=1}^{\infty} 2^{-m}=0,
$$

so that in our present circumstances the series

$$
1+\sum_{m=1}^{\infty} 2^{-m} g\left(2^{m}\right)
$$

can vanish only if $\operatorname{Im} g\left(2^{r}\right)=0,(r=1,2, \cdots)$. The first assertion now follows from the remarks concerning Halász' paper [4] which were made preeceding the statement of Lemma 1, provided we note that uniformly for all integers $N>P$,

$$
\begin{aligned}
& \left|\sum_{p<n \leqq N} n^{-1} \lambda(n)\left(1-\operatorname{Re} g^{*}(n) n^{-i t}\right)-\sum_{p<p \leqq N} p^{-1}\left(1-\operatorname{Re} g(p) p^{-i t}\right)\right| \\
& \quad \leqq \sum_{p} \sum_{k=2}^{\infty} \frac{2}{k p^{k}} \leqq \sum_{p} \frac{1}{p(p-1)}<\infty .
\end{aligned}
$$

In order to prove the second assertion we note that if the nonzero mean-value exists then, (in the notation of the earlier remarks), $C_{0} \neq 0$, so that for some value of $t$ the series

$$
\sum_{n=1}^{\infty} n^{-1} \lambda(n)\left(1-\operatorname{Re} g^{*}(n) n^{-i t}\right)
$$

converges. Moreover, as $x \rightarrow \infty$,

$$
L_{0}(\log x) x^{i t} \rightarrow \mathrm{A} \neq 0,
$$

say.

We next note that we can find an unbounded sequence of positive real numbers $z_{1}, z_{2}, \cdots$ so that $z_{n}^{i t} \rightarrow 1$ as $n \rightarrow \infty$. For, given any positive real number $\varepsilon$ we can apply Dirichlet's theorem on Diophantine approximation (see for example Hardy and Wright [5] pp. 156-157) to deduce that there exists a nonzero integer $m$ so that

$$
\left\|\frac{m t}{2 \pi}\right\|<\varepsilon .
$$

Setting $z=e^{n}$ we see that

$$
\left|z^{i t}-1\right|=|\exp (i m t)-1| \leqq 2 \pi \varepsilon \exp (\varepsilon) .
$$

If $t / 2 \pi$ is irrational our assertion is justified by choosing a sequence of $\varepsilon$ converging to zero. It $t / 2 \pi$ is rational it is clear that we can even choose a sequence $z_{1}, z_{2}, \cdots$ so that $z_{n}^{i t}=1$ holds for all members of the sequence.

It follows that 


$$
L_{0}\left(\log z_{n}\right) \rightarrow A
$$

$n \rightarrow \infty$.

Suppose now that $t \neq 0$. Then because of the slowly-oscillating nature of the function $L_{0}(n)$,

$$
L_{0}\left(\log \left(z_{n} \exp \left(\pi t^{-1}\right)\right)\right) \rightarrow A, \quad(n \rightarrow \infty),
$$

and therefore from (1)

$$
\left(z_{n} \exp \left(\pi t^{-1}\right)\right)^{i t} \rightarrow 1, \quad(n \rightarrow \infty) .
$$

Since by the construction of the $z_{n}$ the left-hand side converges to -1 , we obtain a contradiction. It follows that $t=0$, and that

$$
\lim _{\sigma \rightarrow 1+} \sum_{n=1}^{\infty} \frac{\lambda(n)}{n^{\sigma}}\left(1-g^{*}(n)\right)
$$

exists, and is finite. By a standard Tauberian theorem of Hardy and Littlewood we deduce that the series

$$
\sum_{n=1}^{\infty} n^{-1} \lambda(n)\left(1-g^{*}(n)\right) \text { and } \sum_{p} p^{-1}(1-g(p))
$$

converge.

That these conditions are indeed sufficient follows from Theorem 1 of Halász [4].

This completes the proof of Lemma 1.

Lemma 2. A sequence of distribution functions $F_{n}(z)(\bmod 1)$ $n=1,2, \cdots$ has a limiting distribution $(\bmod 1)$ if and only if for each integer $\nu$

$$
\alpha_{\nu}=\lim _{n \rightarrow \infty} \int_{0}^{1} e^{2 \pi i \nu z} d F_{n}(z)
$$

exists.

Moreover, the limiting distribution, if it exists, is continuous if and only if

$$
N^{-1} \sum_{|\nu| \leqq N}\left|\alpha_{\nu}\right| \rightarrow 0
$$

and absolutely continuous with a derivative which belongs to the class $L^{2}[0,1]$ if and only if the series

$$
\sum_{\nu=-\infty}^{\infty}\left|\alpha_{\nu}\right|^{2}
$$

converges.

Proof. The results of this lemma are well known to workers 
in the field. A proof of the main assertion can be sketched briefly as follows:

The necessity of the condition is clear from integration by parts and an application of Lebesgue's theorem of dominated convergence.

For sufficiency, we note that the sequence $\alpha_{1}, \alpha_{2}, \cdots$ satisfies

$$
\sum_{u=1}^{m} \sum_{v=1}^{m} \alpha_{u-v} z_{u} \bar{z}_{v}=\lim _{n \rightarrow \infty} \int_{0}^{1}\left|\sum_{u=1}^{m} z_{n} e^{2 \pi i u x}\right|^{2} d F_{n}(x) \geqq 0
$$

for all integers $m$ and complex numbers $z_{1}, \cdots, z_{m}$. In the classical terminology it is positive definite. Then by a theorem of Herglotz [6] there is a Borel measure $\mu$ on $[0,1]$, and so a corresponding distribution function $F(x)=\mu[0, x]$, so that

$$
\alpha_{\nu}=\int_{0}^{1} e^{2 \pi i \nu x} d F(x), \quad(\nu=0, \pm 1, \pm 2, \cdots) .
$$

If now $\alpha$ and $\beta$ satisfy $0<\alpha<\beta<1$, then by the stone-Weierstrass theorem the characteristic function of the interval $(\alpha, \beta]$ can be uniformly approximated on the unit interval $0 \leqq x<1$ by polynomials in $\exp (2 \pi i x)$. If $\alpha$ and $\beta$ are points of continuity of $F(x)$ it follows easily from the monotonicity of distribution functions that

$$
F_{n}(\alpha)-F_{n}(\alpha) \rightarrow(F(\beta)-F(\alpha)), \quad(n \rightarrow \infty) .
$$

The second and third results of the lemma are special cases of results from the theory of Fourier series. Both can be found for example, in Edwards [2]. In its present form the assertion concerning the possible continuity of a limiting distribution is due to Wiener.

Proof of Theorem 1. It is clear from Lemma 2 that the distributions

$$
F_{n}(x)=n^{-1} \sum_{\substack{m \leq n \\ f(m) \leqq x(\bmod 1)}} 1
$$

have a limiting distribution $(\bmod 1)$ if and only if the limits

$$
\lim _{n \rightarrow \infty} n^{-1} \sum_{m=1}^{n} e^{\pi i f(m) \nu}=\lim _{n \rightarrow \infty} \int_{0}^{1} e^{2 \pi i \nu x} d F_{n}(x), \quad(\nu=0, \pm 1, \pm 2, \cdots)
$$

exists. We can then apply Lemma 1 to deduce that for each integer $\nu$ one of the following three conditions is to be satisfied:

(i) For each value of $t$ the series

$$
\sum_{p} p^{-1}\left(1-\operatorname{Re}\left(e^{2 \pi i \nu f(p)} p^{-i t}\right)\right)
$$

diverges

(ii) $-1=e^{2 \pi i f(2) \nu}=e^{2 \pi i f\left(2^{2}\right) \nu}=\cdots$ 
(iii) The series

$$
\sum_{p} p^{-1}\left(1-e^{2 \pi i \nu f(p)}\right)
$$

converges.

Of these conditions only the first and third call for comment. Since for each real number $y$

$$
\operatorname{Re}\left(1-e^{2 \pi i y}\right)=-2 \sin ^{2} \pi y=-2(\sin \pi\|y\|)^{2}
$$

and

$$
2 y / \pi \leqq \sin y \leqq y \text { if } 0 \leqq y \leqq \pi / 2,
$$

the first condition is equivalent to the series

$$
\sum p^{-1}\left\|^{\nu} f(p)-t \log p\right\|^{2}
$$

being divergent for each value of $t$.

Likewise, in (iii) the series

$$
\sum_{p} p^{-1}\left(1-\operatorname{Re}\left(e^{2 \pi i \nu f(p)}\right)\right) \text { and } \sum_{p} p^{-1}\|\nu f(p)\|^{2}
$$

converge and diverge together. Moreover, for each real number $y$

$$
|\operatorname{Sin} y-y| \leqq \frac{|y|^{3}}{6}
$$

so that

$$
\begin{aligned}
\operatorname{Sin} 2 \pi \nu f(p) & =\operatorname{Sin} 2 \pi\{\nu f(p)\}=\operatorname{Sin} 2 \pi\|\nu f(p)\| \cdot \operatorname{Sign}\left(\frac{1}{2}-\{\nu f(p)\}\right) \\
& =\operatorname{Sign}\left(\frac{1}{2}-\{\nu f(p)\}\right)\left(2 \pi\|\nu f(p)\|+0\left(\|\nu f(p)\|^{3}\right)\right)
\end{aligned}
$$

and uniformly for all $P>0$

$$
\begin{aligned}
& \left|\sum_{p \leqq P} p^{-1} \operatorname{Sin} 2 \pi \nu f(p)-2 \pi \sum_{p \leqq P} \operatorname{Sign}\left(\frac{1}{2}-\{\nu f(p)\}\right) p^{-1}\|\nu f(p)\|\right| \\
& \quad \leqq \text { constant } \sum_{p} p^{-1}\|\nu f(p)\|^{2} .
\end{aligned}
$$

It is now clear from the previous remark that the series

$$
\sum p^{-1}\left(1-e^{2 \pi i \nu f(p)}\right)
$$

and the pair of series

$$
\sum_{p} p^{-1}\|\nu f(p)\| \operatorname{Sign}\left(\frac{1}{2}-\{\nu f(p)\}\right), \sum p^{-1}\|\nu f(p)\|^{2}
$$

converge and diverge together.

This completes the proof of Theorem 1 .

Proof of Theorem 2. To prove Theorem 2 we prove that uniform- 
ly for all integers $\nu,\left|\alpha_{\nu}\right|$ lies between two positive constant multiples of

$$
\varepsilon_{\nu} \exp \left(-2 \sum_{p} p^{-1} \operatorname{Sin}^{2} \pi \nu f(p)\right)
$$

We note from the remarks preceeding Lemma 1 that if $\alpha_{\nu} \neq 0$ then it has the form

$$
\alpha_{\nu}=\frac{H\left(1+i a_{0}\right)}{1+i a_{0}} \exp \left(-\sum_{n=1}^{\infty} \frac{\lambda(n)}{n}\left(1-g^{*}(n) n^{-i a}{ }_{0}\right)\right)
$$

where

$$
\begin{aligned}
H\left(i+i a_{0}\right) & \\
= & \prod_{p>P}\left(1+\frac{g(p)}{p}+\frac{g\left(p^{2}\right)}{p^{2}}+\cdots\right)\left(1-\frac{g(p)}{p}\right) \prod_{p \leqq P}\left(1+\frac{g(p)}{p}+\cdots\right) \\
= & \prod_{p \leqq P}\left(1+\frac{g(p)}{p}+\cdots\right) \prod_{p>P}\left(1+\frac{f\left(p^{2}\right)-(f(p))}{p^{2}}\right) \\
& \left.+\frac{f\left(p^{3}\right)-f(p) f\left(p^{2}\right)}{p^{3}}+\cdots\right) .
\end{aligned}
$$

It is clear that since $g\left(2^{r}\right) \neq-1$, for every integer $r$ (since $\alpha_{\nu}$ is nonzero),

$$
c_{1} \leqq\left|H\left(1+i a_{0}\right) \varepsilon_{\nu}^{-1}\left(1+i a_{0}\right)^{-1}\right| \leqq c_{2}
$$

for suitable positive constants $c_{1}, c_{2}$ depending at most upon $f(n)$. Moreover,

$$
\left|\sum_{n=1}^{\infty} \frac{\lambda(n)}{n}\left(1-g^{*}(n) n^{-i t}\right)-\sum_{p} \frac{1}{p}\left(1-g^{*}(p) p^{-i t}\right)\right| \leqq 1,
$$

and from these two facts the desired inequalities (2) follow.

If $\alpha_{\nu}=0$ then either $\varepsilon_{\nu}=0$, or $\varepsilon_{\nu} \neq 0$ but

$$
\sum_{p} p^{-1} \operatorname{Sin}^{2} \pi \nu f(p)
$$

diverges, so that with our earlier convention

$$
\exp \left(-\sum_{p} p^{-1} \operatorname{Sin}^{2} \pi \nu f(p)\right)=0
$$

and the inequalities (2) are still valid.

Theorem 2 now follows immediately from Lemma 2. 


\section{REFERENCES}

1. H. Delange, Sur les functions arithmétiques multiplicatives, Ann. Sci. École Norm. Sup. 78 (1961), 273-304.

2. R. E. Edwards, Fourier Series; A Modern Introduction, N. Y. Holt, Rinehard and Winston, 1967.

3. P. Erdös, On the distribution of additive functions, Ann. of Math. 41 (1946), 1-20.

4. G. Halász, Uber die Mittelwerte multiplikativer zahlentheoretischer Funktionen, Acta Math. Acad. Sci. Hung., 19 (1968), 365-403.

5. G. G. Hardy, An Introduction to the Theory of Numbers, E. M. Wright, Oxford, 4th edition 1960 .

6. K. Urbanik, Lectures on Prediction theory, Springer lecture notes, 44, Berlin 1957.

7. H. Weyl, Gleichverteilung von Zahlen mod Eins, Math. Annalen 77 (1916), 313-52.

8. E. Wirsing, Das asyptotische Verhalten von Summen under multiplikative Funktionen II, Acta Math. Acad. Sci. Hung., 18 (1967), 411-467.

Received May 11, 1970.

UNIVERSITY OF COLORADO AND UNIVERSITY OF NOTTINGHAM 



\section{PACIFIC JOURNAL OF MATHEMATICS}

\section{EDITORS}

H. SAMELSON

Stanford University

Stanford, California 94305

C. R. Hовву

University of Washington

Seattle, Washington 98105
J. DUGUNDJI

Department of Mathematics

University of Southern California

Los Angeles, California 90007

RICHARD ARENS

University of California

Los Angeles, California 90024

\section{ASSOCIATE EDITORS}
E. F. BECKENBACH
B. H. NeumanN
F. WOLF
K. YoSHIDA

\section{SUPPORTING INSTITUTIONS}

UNIVERSITY OF BRITISH COLUMBIA

CALIFORNIA INSTITUTE OF TECHNOLOGY

UNIVERSITY OF CALIFORNIA

MONTANA STATE UNIVERSITY

UNIVERSITY OF NEVADA

NEW MEXICO STATE UNIVERSITY

OREGON STATE UNIVERSITY

UNIVERSITY OF OREGON

OSAKA UNIVERSITY

UNIVERSITY OF SOUTHERN CALIFORNIA
STANFORD UNIVERSITY

UNIVERSITY OF TOKYO

UNIVERSITY OF UTAH

WASHINGTON STATE UNIVERSITY

UNIVERSITY OF WASHINGTON

AMERICAN MATHEMATICAL SOCIETY CHEVRON RESEARCH CORPORATION NAVAL WEAPONS CENTER 


\section{Pacific Journal of Mathematics}

\section{Vol. 38, No. $1 \quad$ March, 1971}

Bruce Alan Barnes, Banach algebras which are ideals in a Banach algebra ..... 1

David W. Boyd, Inequalities for positive integral operators............... 9

Lawrence Gerald Brown, Note on the open mapping theorem .............. 25

Stephen Daniel Comer, Representations by algebras of sections over Boolean

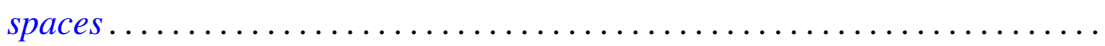

John R. Edwards and Stanley G. Wayment, On the nonequivalence of

conservative Hausdorff methods and Hausdorff moment sequences ........

P. D. T. A. Elliott, On the limiting distribution of additive functions $(\bmod 1) \ldots \ldots$

Mary Rodriguez Embry, Classifying special operators by means of subsets

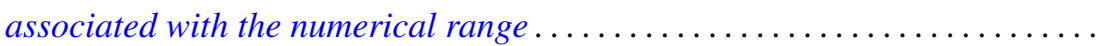

Darald Joe Hartfiel, Counterexamples to a conjecture of G. N. de Oliveira ......

C. Ward Henson, A family of countable homogeneous graphs...............

Satoru Igari and Shigehiko Kuratsubo, A sufficient condition for

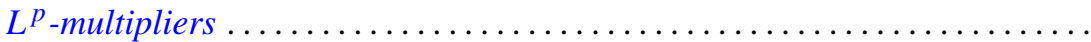

William A. Kirk, Fixed point theorems for nonlinear nonexpansive and

generalized contraction mappings............................

Erwin Kleinfeld, A generalization of commutative and associative rings ...... 95

D. B. Lahiri, Some restricted partition functions. Congruences modulo $11 \ldots \ldots 103$

T. Y. Lin, Homological algebra of stable homotopy ring $\pi *$ of spheres ....... 117

Morris Marden, A representation for the logarithmic derivative of a meromorphic function...........................

John Charles Nichols and James C. Smith, Examples concerning sum properties for metric-dependent dimension functions . .

Asit Baran Raha, On completely Hausdorff-completion of a completely

Hausdorff space.

M. Rajagopalan and Bertram Manuel Schreiber, Ergodic automorphisms and affine transformations of locally compact groups..........

N. V. Rao and Ashoke Kumar Roy, Linear isometries of some function

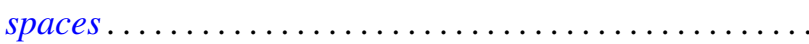

William Francis Reynolds, Blocks and F-class algebras of finite groups

Richard Rochberg, Which linear maps of the disk algebra are multiplicative ...

Gary Sampson, Sharp estimates of convolution transforms in terms of decreasing

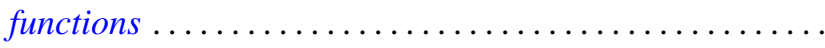

Stephen Scheinberg, Fatou's lemma in normed linear spaces

Ken Shaw, Whittaker constants for entire functions of several complex

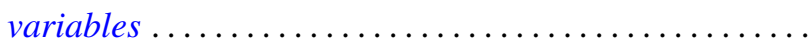

James DeWitt Stein, Two uniform boundedness theorems................ 251

$\mathrm{Li} \mathrm{Pi} \mathrm{Su,} \mathrm{Homomorphisms} \mathrm{of} \mathrm{near-rings} \mathrm{of} \mathrm{continuous} \mathrm{functions} \mathrm{.} \mathrm{.............} 261$

Stephen Willard, Functionally compact spaces, $C$-compact spaces and mappings of minimal Hausdorff spaces....................... 